\title{
Structure Dynamical Phase Transitions Studied by Nuclear (Proton) Magnetic Resonance under Visible and Infrared Irradiation
}

\author{
Kashaev R.S.-H *, Gazizov E.G \\ Kazan State Power Engineering University 420107, Kazan, Esperanto, 62-29 \\ *Corresponding Author: kashaev2007@yandex.ru
}

Copyright (C) 2014 Horizon Research Publishing All rights reserved.

\begin{abstract}
Using pulse nuclear (proton) magnetic resonance relaxation measurements by elaborated relaxometers NMR with irradiation in visual and near infrared range of spectra bitumen and oils were studied. Influence of irradiation on relaxation times and protons populations was investigated. Change of structure-dynamica 1 parameters of molecular motion from temperature was revealed, which are the evidence of molecular motion activation energies $E_{\mathrm{Ai}}$ alteration. At the same time, on the temperature dependences of $T_{2 \mathrm{i}}$ sharp jumps are observed. We attributed them to structure-dynamical phase transitions (SDPT), which appear as a result of formation and subsequent destruction of supermolecular nanodimension structures with temperature growth. Laser irradiation of the sample smoothes or remedy SDPT. NMR-parameters dependences upon NIR irradiation of oil sample, prepared with adding of isoparaffin (dokazan), can be explained by "washing down" of this formed nano dimension structures in terms of energetic levels populations behavior. Time dependences of relaxation time in bitumen sample, which was heated and then cooled in natural conditions, also demonstrate SDPT, which are removed by irradiation.
\end{abstract}

Keywords Structure-Dynamical Phase Transitions, Proton Magnetic Resonance Relaxometry, Irradiation

\section{Introduction}

For express-analysis of high-molecular nano dimensional structures in organic liquids (oils, natural bitumen), existing methods of analysis - near infrared spectroscopy (NIR), $\mathrm{X}$-rays and mass-spectrometry are not fast enough for operative control, because require sample preparation. Nuclear magnetic resonance of high resolution also can not give information about high molecular compounds (HMC) and cannot be used as operative method of control. But optimization of technology processes, prevention accidents and ambient protection require express-control of NHMC in fuels and raw materials. Such opportunities gives nuclear (proton) magnetic resonance relaxometry (NMRR) method, using NMR-relaxometer, which was demonstrated by us at the examples of determination of several physic-chemical properties (PCP) of oils and bitumen [1,2]. NMRR also is unique for slow motions study at associates, especially through spin-spin relaxation times $T_{2 \mathrm{i}}$.

At the same time it was found, that determined by NMRR concentration of components (asphaltens, tars), containing NHMC, differs from concentrations, determined by ISO 3405-88. This is explained by restrictions of molecular motion of NHMC structure fragments and so by shortening of relaxation time below the opportunities of NMR-relaxometer (so called probe head "dead time"). We suggest [3] to "warm up» molecular motion of NHMC fragments by irradiating samples at the wave length of their energy absorbance. This will increase amplitude of molecular motion, and so lead to increase of relaxation times, which give higher sensibility of NMR-relaxometer. The aim of this article is to study NMR-parameters changes due to temperature/time changes at simultaneous irradiation in visual and near infrared (NIR) range of spectra.

\section{Samples and Experimental Setup}

Samples of bitumen and oils were studied. Bitumen has temperature of softening $T_{\mathrm{p}}=48.5{ }^{\circ} \mathrm{C}$, concentrations (in mass $\%$ ) oils - $38.18 \%$, benzol tars $-25.5 \%$, alcohol-benzol tars $-10.53 \%$, asphaltenes $-25.75 \%$, sulphur $\mathrm{S}=3.12 \%$. Petroleum sample, used for samples preparation with different concentrations of isoparaffin have density $\rho=$ $867.5 \mathrm{Kg} / \mathrm{M}^{3}$, kinematic viscosity $v_{20}=42.9 \mathrm{sSt}$, concentration of oils $-51.2 \%$, benzol tars - $17.4 \%$, alcohol-benzol tars- $7.1 \%$, asphaltenes $-3,3 \%$, sulphur $1.6 \%$, waxes $-3.1 \%$. Isoparaffin dokazan $\left(\mathrm{C}_{22} \mathrm{H}_{46}\right)$ have density $\rho=778 \kappa \Gamma / \mathrm{M}^{3}$, melting temperature point $T_{\mathrm{m}}=44^{\circ} \mathrm{C}$, molecular weight 310,6 a.u.

For NMR-relaxation times measurements was used Relaxometer NMR 08/PC on protons resonance frequency $v$ 
$=12.14 \mathrm{MHz}$, elaborated by us according to technical conditions TY 25-4823764.0031-90, and designed portable Relaxometer PMR NP-1 at resonance frequency $v=14.3$ $\mathrm{MHz}$, with autonomous accumulator power supply and control from notebook [4]. Accuracy of single measurement relaxation times was $\pm 3 \%$ relative, signal amplitudes error \pm $2 \%$ relative. Error could be decreased by $n$ accumulations of spin-echo amplitudes. Coefficient of sensibility for diameter $D$ of sample probe is $K=v^{2} D^{3}\left[\mathrm{MHz}^{2} \mathrm{~cm}^{3}\right]$. For our NMR-relaxometers it is $K=2700-4150 \mathrm{MHz}^{2} \mathrm{~cm}^{3}$, that is near to $K=1600-6400 \mathrm{MHz}^{2} \mathrm{~cm}^{3}$ of laboratory NMR-relaxometer "Minispec pc120" (Bruker, Germany). For temperature measurements was used temperature sample probe [5], which stabilized temperature with accuracy \pm 0.5 ${ }^{\circ} \mathrm{C}$ in the temperature range $20-+200{ }^{\circ} \mathrm{C}$. Spin-spin relaxation times $T_{2 \mathrm{i}}$ and proton populations $P_{\mathrm{i}}$ were measured using Carr-Purcell-Meiboom-Gill (CPMG) pulse sequence $90^{\circ}-\tau-\left(180^{\circ}-2 \tau-\right)_{\mathrm{N}}$ with parameters: period of sequence starts $T=2$ c, number of $180^{\circ}$ pulses $N=100 \div 1000$, interval between $180^{\circ}$ pulses $2 \tau=200 \mu$ s, amplification $12-27$, number of accumulations $n=10 \div 100$.

As a source of visible (red) coherent radiation the He-Ne medical laser "ЛНГ-111" with radiation wave length $\lambda=$ $0.63 \pm 0.05 \mu \mathrm{m}$ was used. Spectral composition of radiation manymodal, power $-25 \mathrm{~mW}$, diameter of laser beam $-4 \mathrm{MM}$, relative stability of radiation power (during 8 hours) $-2 \%$, consumed power $-100 \mathrm{~W}$. As a NIR radiation at $\lambda=1.825$ $\mu \mathrm{m}$ was used a source, consisting from lamp ТРШ 1500-2300 (TC 16.535.847-74) and three-layered, interferential light filters with range $30 \mathrm{~nm}$.

\section{Experimental Results}

As it is known, energy $E_{\mathrm{i}}$ of the molecule is a sum of electronic energy and energies of oscillation, rotation and translation types of motion. After electromagnetic radiation absorption, energy pass from $i$ level to more high $j$ level with change its value:

$$
\Delta E_{\mathrm{ij}}=E_{\mathrm{i}}-E_{\mathrm{j}}=h v=h c / \lambda \sim 1.2 \cdot 10^{5} / \lambda
$$

where $\Delta E_{\mathrm{ij}}$ in $\mathrm{KJ} / \mathrm{mole}$, wave length $\lambda$ - in $\mathrm{nm}, h=6.62 \cdot 10^{-34}$ $\mathrm{J} \cdot \mathrm{s}-$ Plank constant. Oscillating motions are the most variant and every hydrocarbon has definite strip of absorbance in spectra. So secondary oil aromatic residues absorb in visible range of spectra at $0.434-0.7 \mu \mathrm{m}$. 1.87-2.1 $\mu \mathrm{m}$ - range of absorption of waxes in petroleum and petroleum products. It leads to excitation of the deformation and valence oscillations of molecular fragments.

Effect of irradiation is well known. In medicine lasers on $\lambda$ $=0.63 \mu \mathrm{m}$ are used and in spite of low power of irradiation $(\sim$ $20 \mathrm{~mW}$ ), they are effective for selective excitation of blood components. In analogy, exciting (by irradiation) definite molecular motions (warming up oscillating modes), we can selectively activate definite molecules with their characteristic set of molecular oscillations ( $i$-th proton phases), having their spin-spin relaxation times $T_{2 \mathrm{i}}$. As a result of irradiation $T_{2 \mathrm{i}}$ will arise on $\Delta T_{2 \mathrm{i}}{ }^{*}$ to higher $T_{2 \mathrm{i}}{ }^{*}$ values, because intermolecular relaxation rate $\left(T_{2 \mathrm{i}}\right)^{-1}$ depends from $\tau_{\mathrm{B}}-$ correlation times of rotation motion (time of life in the definite position) and $r_{\mathrm{ij}}$ - inter proton distance by equation [6]:

$$
\left(T_{1,2 \mathrm{~B}}\right)^{-1}=3 \gamma^{4} h^{2} \tau_{\mathrm{B}} / 4 \pi^{2} r_{\mathrm{ij}}{ }^{6}
$$

where $\gamma=2.675 \cdot 10^{4} \mathrm{rad} . / \mathrm{sec} \cdot \mathrm{oe}$ - hyromagnetic ratio for protons. Excitation of molecular rotations by irradiation lead to decrease of $\tau_{\mathrm{B}}$ and to increase of mean inter proton distances $r_{\mathrm{ij}}$. As a result relaxation times $T_{2 \mathrm{i}}$ increase up to $T_{2 \mathrm{i}}{ }^{*}$ values.

Envelope of spin-echo amplitudes $A_{\mathrm{e}}$ decay in the CPMG-method can be described by the equation:

$$
\begin{gathered}
\mathrm{Ae}=\sum \mathrm{A} 0 \mathrm{i} \exp (-\mathrm{t} / \mathrm{T} 2 \mathrm{i}) \\
\mathrm{i}=\mathrm{A}, \mathrm{B}, \mathrm{C}
\end{gathered}
$$

where $A_{0 \mathrm{i}}$ (in arbitrary units) correspond to proton populations $P_{\mathrm{A}}, P_{\mathrm{B}}$ and $P_{\mathrm{C}}, T_{2 \mathrm{i}}$ - spin-spin relaxation times $T_{2 \mathrm{~A}}, T_{2 \mathrm{~B}}$ and $T_{2 \mathrm{C}}$, corespond to proton phases $i=A, B$ and $C$. This phases conditionally can be attributed: $A$ - to dispersion medium of light paraffins and naftens with highest mobility of $\mathrm{CH}_{3}$ - and $\mathrm{CH}_{2}$-chains; $B$ - to the resins (tars); $\mathrm{C}-$ to asphaltenes. Conditionality of attribution is caused by the circumstance, that some high mobile molecular fragments of $B$ and $C$ phases can contribute to relaxation time $T_{2 \mathrm{~A}}$ of phase $A$ and vice versa, some low mobile fragments of phase $A$ can attribute to relaxation times $T_{2 \mathrm{~B}}$ and $T_{2 \mathrm{C}}$. But on the whole populations approximately characterize concentrations of paraffin-naftenes, resins (tars) and asphaltenes.

In science literature for description of the disperse phase element in oils was accepted the term "structure unit" (SU) [7]. SU are phase fractal particles, which can be described on the basis of dynamical phenomenon of the fractal cluster, composed from asphaltene (or paraffin) core and solvate envelope. Fractal cluster is sedimented in the medium of light hydrocarbons.

Obtained results are presented at fig.1-5. As it is seen from fig.1, with the temperature growth, values of spin-spin relaxation times $T_{2 \mathrm{~A}}, T_{2 \mathrm{~B}}$ and $T_{2 \mathrm{C}}$ in bitumen arise. Graphs are characterized by slopes, changing from temperature. This is the evidence of the activation energy $E_{\mathrm{Ai}}$ of molecular motions change. In particular, with the temperature growth, the slope decrease and so decreases mean value of activation energies $E_{\mathrm{Acp}}$, calculated using formula (4) and assuming mainly intra molecular contribution $\left(T_{1,2 \mathrm{~B}}\right)^{-1}$ in relaxation of oscillating and rotating side $\mathrm{CH}_{2}$ and end $\mathrm{CH}_{3}$-groups. In [8] it was shown, that eqs. $(2,4)$ are valid, if intermolecular contribution in relaxation from translation diffusion in order of value is lower than intra molecular contribution from oscillation and rotation motions of molecular fragments. That is so in oil disperse systems of bitumen and petroleum. Arrenius dependence for correlation times of $\mathrm{CH}_{3}$ - and $\mathrm{CH}_{2}$-chains rotation motions was assumed with $\tau_{\mathrm{C}}=$ $\tau_{0} \exp \left(E_{\mathrm{A}} / \mathrm{R} T\right)$, where $\tau_{\mathrm{o}}$ - pre-exponential factor, $\left(\tau_{0}\right)^{-1}$ indicating frequency of proton oscillations in definite position, $R=8,314 \mathrm{~J} /{ }^{\circ} \mathrm{K} \cdot \mathrm{mole}, T$ - absolute temperature. 
Quantity of $E_{\mathrm{A}}$ is calculated using equation from [6]:

$$
E_{\mathrm{A}}=19,13 \lg \left(T_{2}^{(2)} / T_{2}^{(1)}\right)\left[T^{(1)} T^{(2)} /\left(T^{(2)}-T^{(1)}\right)\right]
$$

where $T_{2}^{(2)}, T_{2}^{(1)}$ - spin-spin relaxation times, measured at $T^{(1)}$ and $T^{(2)}$ temperatures.

It was established, that with temperature growth $E_{\mathrm{A}}$ drop. So, for temperature interval $10^{3} / T=2.9-3.4$, our calculated $E_{\mathrm{A}}=43.89 \mathrm{~kJ} / \mathrm{mole}$, for $10^{3} / T=2.78-2.9, E_{\mathrm{A}}=20.48 \mathrm{~kJ} / \mathrm{mole}$, for $10^{3} / T=2.6-2.78, E_{\mathrm{A}}=16.72 \mathrm{~kJ} / \mathrm{mole}$. Such drop of $E_{\mathrm{A}}$ can be explained by removal of molecular motion restrictions with temperature growth. At the same time, the spin-spin relaxation times dependences demonstrate sharp changes of $T_{2 \mathrm{i}}$, which can be explained by phase transitions, named by us "structure-dynamical phase transitions" (SDPT), which, probably, arise as a result of temporal (dynamical) super molecular structure units (SU) formation and the following their destruction with temperature growth. In our case SDPT are due to SU fractal structure ordering, accompanied by effective SU core diameter decrease (decrease of $P_{\mathrm{C}}$ proton population) and solvate envelope thickness increase (increase of $P_{\mathrm{B}}$ ). Changes of $T_{2 \mathrm{C}}, T_{2 \mathrm{~B}}$ at SDPT can be explained by eq. (2) as a decrease of inter proton mean distances $r_{\mathrm{ij}}$ due to structure ordering of molecules in asphalten core and near core solvate cover at filling of fractal space. Changes of core and solvate cover under temperature and pressure affect are described also in [7].

At SDPT temperatures we observe negative slope $T_{2 \mathrm{i}}(1 / T$ $K)$ of relaxation times with temperature rise and hence negative activation energies $E_{\mathrm{Ai}}$. This means, that process goes exothermically, with emission of heat, that is common for ordering processes. So SDPT is probably caused by structure ordering of aliphatic chains and rings of aromatic and naphtens/resins at SU core cover (envelope). It is an aspiration of open dissipative systems to minimum of free energy and to decrease of entropy production [9]. Laser irradiation smooth SDPT (curves 4-6 at fig.1). That effect can be explained by destruction of super molecular structures of SU as a result of light radiation energy absorption by aromatic fragments of ashaltens and resins, which include 4-5 aromatic rings, absorbing radiation at $\lambda=0.63 \pm 0.05$ $\mu \mathrm{m}$.

$T_{2 \mathrm{~A}}, T_{2 \mathrm{~B}}, T_{2 \mathrm{C}}(\mathrm{ms})$

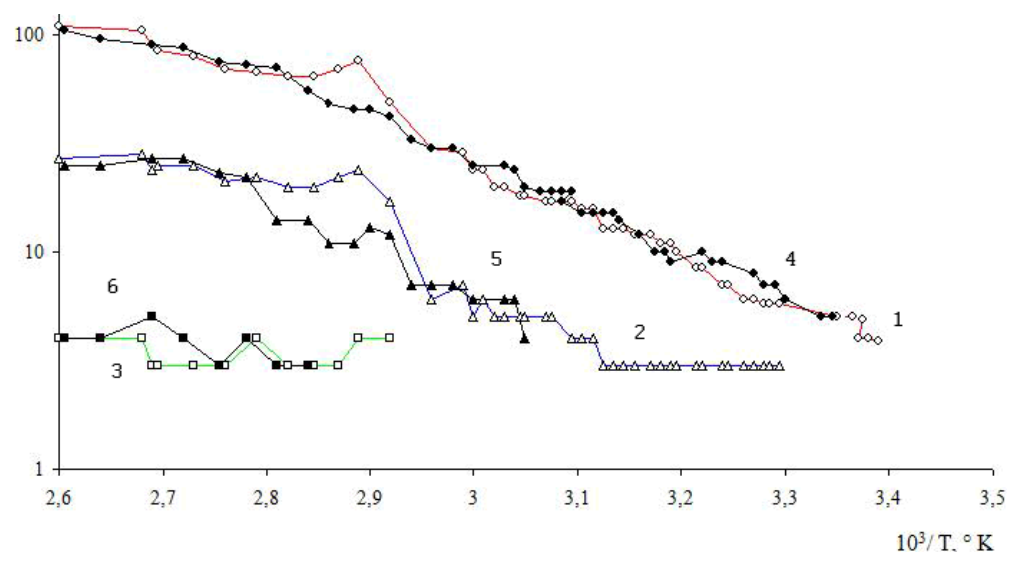

Figure 1. Dependences of spin-spin relaxation times T2i (ms) in bitumen from reverse temperature 103/T K . Curves 1-3 - T2A, T2B, T2C without irradiation, curves 4-6 - the same at irradiation by He-Ne laser at wavelength $\lambda=633 \mathrm{~nm}$.

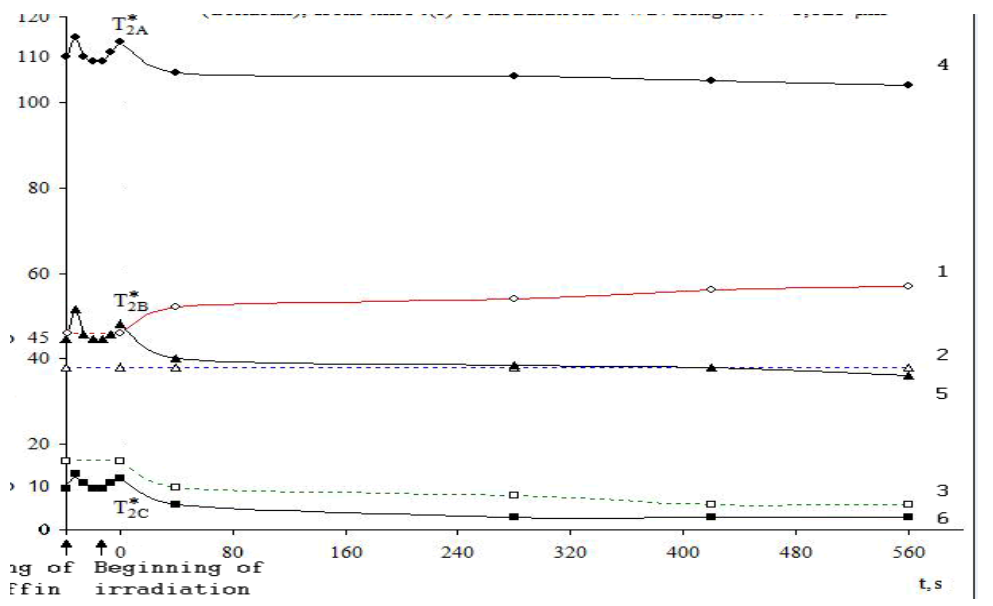

Figure 2. Dependences of spin-spin relaxation times $\mathrm{T} 2 \mathrm{i}(\mathrm{ms})$ and proton populations $\mathrm{Pi}(\%)$ in petroleum , prepared with adding of paraffin (dokasan), from time $\mathrm{t}(\mathrm{s})$ of irradiation at wavelength $\lambda=1,825 \mu \mathrm{m}$. Curves $1-3-\mathrm{T} 2 \mathrm{~A}, \mathrm{~T} 2 \mathrm{~B}, \mathrm{~T} 2 \mathrm{C}$, curves 4-6 - PA, PB, PC. 
At fig. 2 values of spin-spin relaxation times are presented as a dependence of irradiation time $t$ in near infrared (NIR) range of spectra at $\lambda=1.825 \mu \mathrm{m}$. In studied oil sample $1 \mathrm{~g}$ of dokazan was added with the aim to create conditions for SU formation on the basis of isoparaffin molecules. As it is seen from fig.2, as a result the following processes here take place:

1) after dissolution of isoparaffin in oil, relaxation times decrease $T_{2 \mathrm{~A}}$ on $4 \mathrm{~ms}$ and $T_{2 \mathrm{~B}}$ on $6 \mathrm{~ms}$;

2) at the initial stage of irradiation at the first seconds relaxation times $T_{2 \mathrm{~A}} *, T_{2 \mathrm{~B}} *, T_{2 \mathrm{C}} *$ of irradiated sample increase on $\Delta T_{2 \mathrm{~A}} *=3.6 \%, \Delta T_{2 \mathrm{~B}} *=9 \%$ and $\Delta T_{2 \mathrm{C}} *=$ $15 \%$;

3) during irradiation the $T_{2 \mathrm{i}}$ * relaxation times decrease, governing by dependences:

$$
\begin{gathered}
T_{2 \mathrm{~A}}{ }^{*}=109 \exp \left(-t / 1,35 \cdot 10^{4}\right)+40 \exp (-t / 23) \\
T_{2 \mathrm{~B}}{ }^{*}=40,5 \exp \left(-t / 1,29 \cdot 10^{4}\right)+8 \exp (-t / 20) \\
T_{2 \mathrm{C}}{ }^{*}=4,8 \exp (-t / 840)+1,55 \exp (-t / 100)++4,7 \exp (-t / 22)
\end{gathered}
$$

4) proton populations Pi demonstrate arise of PA and decrease of $\mathrm{PC}$ during the irradiation process, which can be described by equations:

$$
\begin{array}{r}
P_{\mathrm{A}}(\%)=100-49 \exp (-t / 3600)-45 \exp (-t / 20) \\
P_{\mathrm{C}}(\%)=10,5 \exp (-t / 960)+5 \exp (-t / 30)
\end{array}
$$

This processes of dynamical changes of NMR-parameters $\left(T_{2 \mathrm{i}}{ }^{*}\right.$ and $\left.P_{\mathrm{i}}\right)$ can be explained by:

1) initial small increase before irradiation is caused by solid dokazan absorption of molecules from oil and so lowering of media total viscosity. As it was earlier established by us relaxation times and viscosity are in inverse proportional dependence [1] and for instance for $A$ phase

$$
\left[\eta\left(T_{2 \mathrm{~A}}\right)^{3 / 2}\right] / T=78.3
$$

The following sufficient decrease of relaxation times is caused we thing by dokozan solution and SU formation around paraffin;

2) relaxation times $T 2 A$ and $T 2 B$ increase at initial stage of irradiation is caused by intensification of molecular motion of molecular fragments in proton phases A, B and $\mathrm{C}$ according to eq.(2) as a result of NIR-radiation absorption at $\lambda=1.825 \pm 0.03 \mu \mathrm{m}$ (absorb paraffin-naphtens and aliphatic chains of asphaltens and resins (tars) in SU);

3) subsequent decrease of $T 2 \mathrm{~A}^{*}, \mathrm{~T} 2 \mathrm{~B}^{*}$ and $\mathrm{T} 2 \mathrm{C}^{*}$ at continuous irradiation can be explained as a result of restructuring of SU and SU core/envelope ordering through colloid gel-structure formation. This lead to higher viscosity according to eq.(10). It should be also mentioned, that process goes in two and three stages (eq.5-7) for phases A and B and C with different time constants;

4) increase of PA and decrease of PC (fig.2) at irradiation confirm point 3 ) and is the witness of SU restructuring with gel-structure formation in disperse medium with decrease of phase $\mathrm{C}$ protons population and increase of protons population of phase $\mathrm{A}$.

The increments $\Delta T_{2 \mathrm{~A}}{ }^{*}, \Delta T_{2 \mathrm{~B}}{ }^{*}$ and $\Delta T_{2 \mathrm{C}} *$ to relaxation times at irradiation can find explanation in terms of phenomenological description of energy levels (see for instance [10]). Change of NMR-parameters during NIR irradiation of oil sample can be explained in the terms of energetic levels populations behavior.

Irradiation affect we will take into consideration using three-level system of quantum amplifier (maser), where levels 1 and 2 - with populations $n_{1}$ and $n_{2}$-result of Zeeman interaction of spin system with external magnetic field $B_{0}$ with condition $n_{1}+n_{2}+n_{3}=N$, where $n_{3}$ corresponds to level of optical pumping:

$$
E_{\mathrm{m}}=-\gamma h m B_{0} / 2 \pi
$$

where quantum number $m= \pm 1 / 2$. This is the system, where difference of inverse population of first and second levels is approached due to saturation of the third level by optical or NIR pumping. Populations can change as result of relaxation with times:

$$
T_{1 \mathrm{ik}}{ }^{-1}=w_{\mathrm{ik}}+w_{\mathrm{ki}}
$$

where $w_{\mathrm{ik}}, w_{\mathrm{ki}}$ - probabilities of relaxation transitions, while $w_{\mathrm{ik}} \neq w_{\mathrm{ki}}$, value of $w_{\mathrm{ik}}$ depends from temperature $w_{\mathrm{ik}}=$ $w_{\mathrm{ki}} \exp \left(h v_{\mathrm{ik}} / k T\right)$. Changes of 1 and 3 levels populations under irradiation are described by $W_{13}\left(W_{13}=W_{31}\right)$. Dynamic of levels populations change under continuous irradiation can be described by equations:

$$
\begin{gathered}
d n_{1} / d t=-\left(w_{12}+w_{13}\right) n_{1}+w_{21} n_{2}+w_{31} n_{3}-\left(n_{1}-n_{3}\right) W_{13} \\
d n_{2} / d t=-\left(w_{12}+w_{23}\right) n_{2}+w_{12} n_{1}+w_{32} n_{3}-\left(n_{2}-n_{3}\right) W_{13} \\
d n_{3} / d t=-(1 / 2)\left[\left(w_{31}+w_{32}\right) n_{3}\right]-\left(n_{3}-n_{1}\right) W_{13}
\end{gathered}
$$

System can be rewritten in the form:

$$
\begin{gathered}
d n_{1} / d t=-(1 / 2)\left[\left(T_{12}{ }^{-1}+T_{13}{ }^{-1}\right) n_{1}+T_{21}{ }^{-1} n_{2}+T_{31}{ }^{-1} n_{3}\right] \\
\left.n_{3}\right) W_{13}
\end{gathered}
$$

so:

$$
\begin{aligned}
& d \Delta n_{12} / d t=d n_{1} / d t-d n_{2} / d t=-(1 / 2)\left[\left(T_{13}{ }^{-1} n_{1}+T_{23}{ }^{-1} n_{2}\right]-\left(n_{1}-\right.\right. \\
& \left.n_{2}\right) W_{13}
\end{aligned}
$$

Solution of equation (19) for magnetization $M(t)_{\text {irr }}$ growth will be:

$$
M(t)_{\text {irr }}=M(0) \exp \left\{-(1 / 2)\left[\left(T_{13}{ }^{-1} n_{1}+T_{23}{ }^{-1} n_{2}\right]-\left(n_{1}-n_{2}\right) W_{13}\right\}\right.
$$

So, to the relaxation time of magnetization decay $M(t)=$ $M_{\perp}(t)=M(0) \exp \left(-t / T_{2}\right)$ we must add time for optical pumping of third level 3 and for $2 \rightarrow 3$ transitions. This will lead to $T_{2}$ increase. Such effect with $\Delta T_{2 \mathrm{~A}}, \Delta T_{2 \mathrm{~B}}$ and $\Delta T_{2 \mathrm{C}}$ we observe at fig. 2 at the first minute of irradiation. 


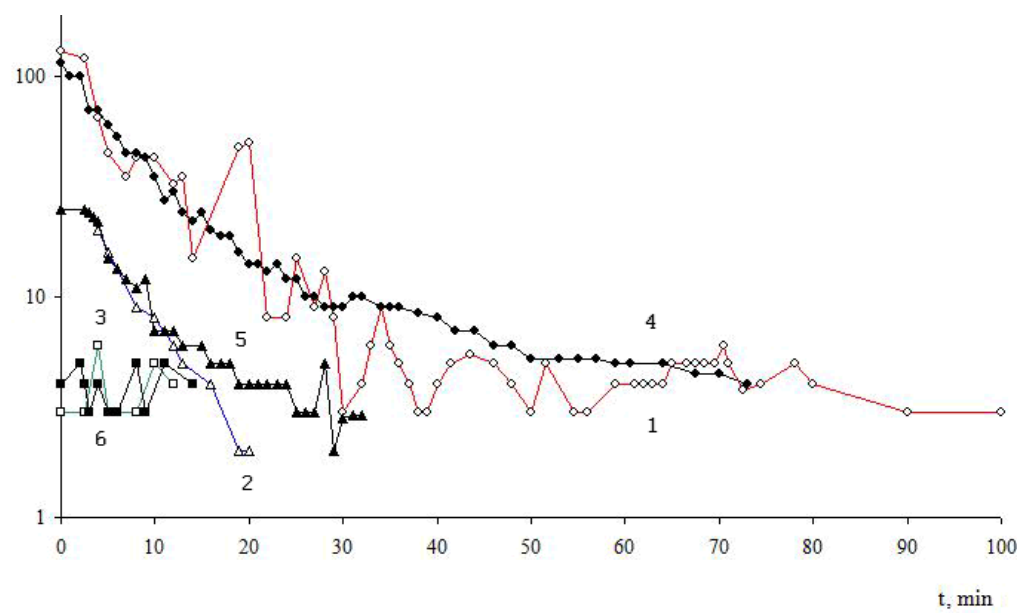

Figure 3. Dependences of spin-spin relaxation times $\mathrm{T} 2 \mathrm{i}(\mathrm{ms})$ in bitumen from time of cooling. Curves 1-3 - T2A, T2B, T2C without irradiation, curves 4-6 - the same at irradiation by He-Ne laser at wavelength $\lambda=633 \mathrm{~nm}$

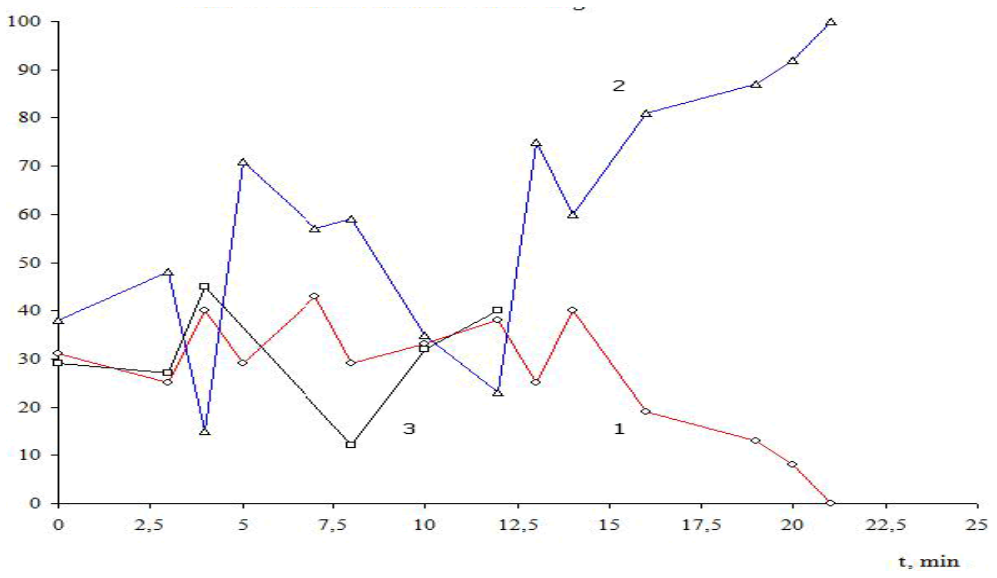

Figure 4. Time dependences of proton populations Pi (\%) (curves 1-3 - PA, PB, PC.) in bitumen without irradiation

On fig. 3 temporal dependence of relaxation times $T_{2 \mathrm{~A}}$ and $T_{2 \mathrm{C}}$ in natural sample of bitumen, heated to $110^{\circ} \mathrm{C}$ and cooled in natural conditions (room temperature $20{ }^{\circ} \mathrm{C}$ ), manifest sharp chances of relaxation times. We attributed them also to structure-dynamical phase transitions (SDPT), which appear as a result of formation and destruction with temperature of super molecular structures in bitumen. Laser irradiation of the bitumen sample smoothes them.

\section{$T_{2 \mathrm{i}}(\mathrm{ms})$}

Temporal changes of $T_{2 \mathrm{~A}}, T_{2 \mathrm{~B}}$ in not radiated and $T_{2 \mathrm{~A}}{ }^{*}, T_{2 \mathrm{~B}}{ }^{*}$ in irradiated at the wave length $\lambda=1,825 \mu \mathrm{m}$ sample at cooling can be approximately described by equations:

$$
\begin{array}{r}
T_{2 \mathrm{~A}}=\cos (\alpha t)[\exp (-t / 6000)+1,4 \cos (\alpha t) \exp (-t / 480)] \\
T_{2 \mathrm{~A}}{ }^{*}=\exp (-t / 6000)+11,4 \cos (\alpha t) \exp (-t / 480) \\
T_{2 \mathrm{~B}}=3,3 \exp (-t / 360) \\
T_{2 \mathrm{~B}}{ }^{*}=0,9 \exp (-t / 1500)+2,4 \exp (-t / 270)
\end{array}
$$

where $\alpha \approx 510$. For $T_{2 \mathrm{C}}$ analytical approximation was not done because this phase has very short existence time interval and not sufficient precision of relaxation times determination.

Time dependences of relaxation time in not radiated bitumen sample, demonstrating sharp chances of relaxation times (eq.21) and attributed by us to SDPT, are smoothed at irradiation. This SDPT - are phase transition, which cannot be ascribed neither to phase transitions (PT) of I order, nor to PT of II order. Really, according to definition, the PTII must be accompanied by jump of second derivative of thermo dynamical potential without any heat emission, exothermic effect which takes place at PTI. But we observe such effect directly through temperature increases at SDPT temperatures as seen at fig 5. Decrease of $T_{2 \mathrm{~A}}$ at SDPT at fig 3 is accompanied by decrease of temperature (fig 5), that is connected with heat absorption from bitumen system, needed for structure transformation with ordering of new temporal fractal superstructure. It should be mentioned, that temperature measurements were accomplished with precision $0,5^{\circ}$ and only from 45 -th minute of irradiation in view of sufficient temperature noise at high temperatures, which smooth temperature changes. SDPT at high temperatures are confirmed by proton populations dependences for $A, B$ and $C$ phases at fig. 4. Here minimums of $T_{2 \mathrm{~A}}$ coincide with maximums of $P_{\mathrm{A}}$ (temporal increase of phase $A$ density), probably because of protons delay from phase $B\left(P_{\mathrm{B}}\right.$ has minimum). Laser irradiation at $\lambda=633 \mathrm{~nm}$ smooth this effect and remove SDPTs.

$$
P_{\mathrm{i}}(\%)
$$




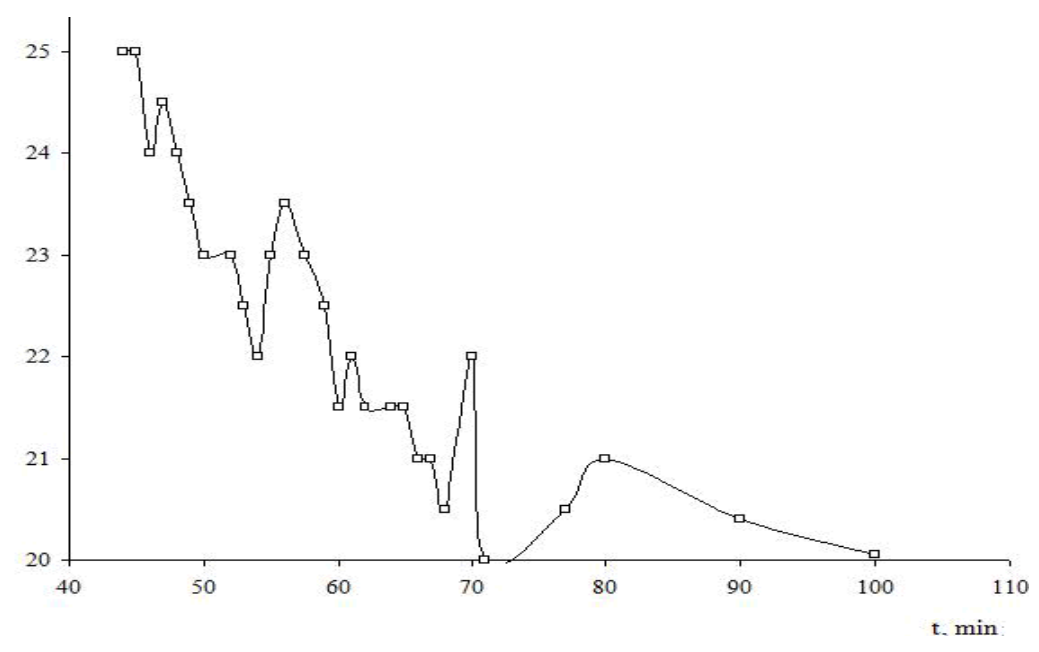

Figure 5. Time dependences of temperature $\mathrm{T}$ oC in bitumen without irradiation

\section{Conclusions}

For all the samples envelope of spin-echo amplitudes has at least three components with spin-spin relaxation times $T_{2 \mathrm{~A}}$, $T_{2 \mathrm{~B}}$ and $T_{2 \mathrm{C}}$, coresponding to proton phases $i=A, B$ and $C$ with populations $P_{\mathrm{A}}, P_{\mathrm{B}}$ and $P_{\mathrm{C}}$. Slope of relaxation times temperature dependence changes from temperature measuring interval. This is the evidence of molecular motion activation energies $E_{\mathrm{Ai}}$ changes - decrease with temperature growth. At the same time, on the temperature dependences sufficient chances of spin-spin relaxation times are manifested. We attributed them to structure-dynamical phase transitions (SDPT), which appear as a result of formation and destruction with temperature growth of supermolecular nano dimension structures. Laser irradiation of the sample smoothes SDFT, that can be explained by structure units destruction as a result of exciting of aromatic chaines oscillations. Changes of NMR-parameters during NIR irradiation of oil sample, prepared with the adding in the oil of paraffin (dokasan), at initial times are caused by exciting of molecular motions, which can be theoretically explained in the terms of energetic levels populations behavior. Time dependences of relaxation time in bitumen sample, heated and then cooled in natural conditions, also demonstrate in nonradiated sample the SDPT, confirmed by exothermic effects and protons populations behavior. They are also smoothed by irradiation.

\section{REFERENCES}

[1] Kashaev R.S., (2001) "Scientific bases of structure

[2] Kashaev R.S., Chairullina I.R., (2009), "The Influence of Sulfur on the Structural

[3] Kashaev R.S., (2009) Patent of Russian Federation \# $2009138707 / 19$

[4] Idijatullin Z.Sh., Temnikov A.N., Kashaev R.S., (2007)Portable relaxometer of Nuclear Magnetic Resonance Patent of RF № 67719.

[5] Idijatullin Z.Sh., Kashaev R.S., Temnikov A.N. (2006) Patent of RF on invention № 2319138.

[6] Vashman A.A., Pronin I.S., (1979) "Nuclear Magnetic Relaxation and its application in chemical physic", Moscow, "Science" publishing,

[7] Safieva R.Z., (1998), "Physic

[8] Frolov V.V. (1969), "Models of molecular motion in proton relaxation theory in fluids", Collection "Nuclear Magnetic Resonance". St.Petersb.: SPU. III.

[9] Kuseev I.R. (1987), "Perfection of technology and long

[10] Goldman M. (1988), "Quantum description of high 